\title{
Limited evidence on the best position for prosthetic margins
}

\section{Abstracted from \\ Papageorgiou SN, Papadelli AP, Koidis PT, Petridis HP.}

The effect of prosthetic margin location on caries susceptibility. A systematic review and meta-analysis. Br Dent J 2013; 214: 617-624. doi: 10.1038/sj.bdj.2013.582. PubMed PMID: 23787852.

Address for correspondence: Dr. Haralampos P. Petridis, Department of Restorative Dentistry, Prosthodontics Unit, UCL Eastman Dental Institute, London, UK. E-mail: c.petridis@ucl.ac.uk

\section{Question: Does prosthetic margin placement have an influence on caries susceptibility?}

Data sources The Cochrane Central Register of Controlled Trials (CENTRAL), Cochrane Database of Systematic reviews, Medline, Google Scholar and Scopus databases were searched along with handsearching of the Journal of Prosthetic Dentistry, International Journal of Prosthodontics, Journal of Dentistry and Caries Research. Study selection Randomised controlled clinical trials, prospective or retrospective studies or case-control observational studies reporting on caries susceptibility of teeth serving as abutments for prosthetic restorations were included. Only English language studies were included. Data extraction and synthesis Study selection and quality assessment was carried out by two reviewers. Trials were compared by grouping abutments teeth/abutment surfaces with subgingival margins. Caries incidence was evaluated as a dichotomous variable. The risk ratio (RR) for caries incidence of prosthetic margins was calculated for the direct comparisons and pooled using a random-effects model. The overall quality of evidence supporting the association between secondary caries and margin placement was assessed using the GRADE system. Results Twenty-two studies were included, representing 2,648 prosthetic restorations placed in 1,242 patients with mean follow-up time ranging from two to 11.4 years. A random effects meta-analysis was performed on two studies. This found a pooled risk ratio (RR) for secondary caries of sub-gingival margins at up to five years of 1.25 ( $95 \% \mathrm{Cl}: 0.70$ to 2.22 ). At ten years of follow-up, the RR was 1.22 (95\% Cl: 0.81 to 1.83 ) and at 15 years 0.67 ( $95 \% \mathrm{Cl}: 0.45$ to 1.00 ).

Conclusions This systematic review and meta-analysis failed to detect a significantly different secondary caries rate of subgingivally located prosthetic margins in the short to mid-term (10 years). Due to the small number and the limitations of the included studies the results do not provide conclusive evidence as to the effect of prosthetic margin placement on the incidence of secondary caries.

\section{Commentary}

Cast prosthetic restoration in private practice is reported to have a ten year survival rate of over 90\%. ${ }^{1}$ Despite their impressive prognosis, cast prosthetic restorations occasionally fail, typically as a result of caries. ${ }^{2,3}$ Dental caries progression is conventionally managed with fluoride exposure and diet control. However, it is theorised that dental restoration margins below the gingival crest (Sub-G margins) are protective against caries attack because the biochemical environment in the gingival sulcus - anaerobic, alkaline and void of dietary components - inhibits the activity of carious causing bacteria. ${ }^{4}$

The objective of Papagreorgiou et al.'s review ${ }^{4}$ was to answer the following clinical question: are sub-gingival prosthetic margins less susceptible to secondary caries than prosthetic margins at or above the gingival crevice. This review followed a standardised protocol (PRISMA statement). The search strategy was thorough with clearly defined inclusion and exclusion criteria. This led to a generally impressive agreement between the two reviewers (Kappa; 0.7150.933). However, the search was limited to only English articles and included both prospective and retrospective studies. Ideally, such an intervention-based clinical question would best be answered with randomised controlled trials (RCTs), which may have been published in a language other than English.

Only two of the 22 included articles were prospective studies, reflecting the generally low quality of studies of which this review was comprised. Also, a large majority of the included prostheses $(83.9 \%)$ had margins at or above the gingival margins. This imbalance between the two groups is largely due to the fact that margin locations were determined at the end of the study. This is significant because the progression of gingival recession around prosthetic margins is a common occurrence. ${ }^{5}$ Furthermore, significant variation existed in how marginal caries were measured and assessed between included studies. Sub-G crown margins are more difficult to identify visually or tactilely with an explorer. As well, radiographs are unreliable at assessing mild to moderate marginal caries.

The five years pooled risk ratio favoured prosthetic margins at or above the gingival crevice with a $\mathrm{RR}=1.25$ (95\%CI-0.70-2.22) at the surface level. But this was based on only two generally poor quality studies (141 patients) with a dropout rate of $40 \% .{ }^{6,7}$ The derived ten and 15 years pooled risk ratio estimates are based on only one small study (108 patients) with a dropout rate of $45 \%$. Such a large dropout rate threatens the validity of the evidence generated from this review. Interestingly, the pooled 15 years risk ratio favoured 


\section{RESTORATIVE DENTISTRY}

the Sug-G prosthetic margin group with an RR of 0.67 (95\%CI: 0.45 -1.00). However, statistical significance between the two groups was not reached at five, ten or 15 years.

In conclusion, due to the dearth of quality and quantity in the available evidence, it is not possible to come to a conclusion on the caries protective nature of Sub-G prosthetic margins. However, there is evidence demonstrating the risk of harm that Sub-G prosthetic margins have on the periodontal apparatus compared to prosthetic margins at or above the gingival crevice. $5,7-10$

Ben Balevi

University of British Columbia, Vancouver, Canada

1. Reitemeier B, Hänsel K, Kastner C, Weber A, Walter MH. A prospective 10-year study of metal ceramic single crowns and fixed dental prosthesis retainers in private practice settings. J Prosthet Dent 2013; 109: 149-155

2. Sailer I, Pjetursson BE, Zwahlen $\mathrm{M}$, Hämmerle $\mathrm{CH}$. A systematic review of the survival and complication rates of all-ceramic and metal-ceramic reconstructions after an observation period of at least 3 years. Part II: Fixed dental prostheses. Clin Oral Implants Res 2007; 18 Suppl 3: 86-96.

3. Libby G, Arcuri MR, LaVelle WE, Hebl L. Longevity of fixed partial dentures. J Prosthet Dent 1997; 78: 127-131.

4. Papageorgiou SN, Papadelli AP, Koidis PT, Petridis HP. The effect of prosthetic margin location on caries susceptibility. A systematic review and meta-analysis. Br Dent J 2013; 214: 617-624

5. Bader JD, Rozier RG, McFall WT Jr, Ramsey DL. Effect of crown margins on periodontal conditions in regularly attending patients. / Prosthet Dent 1991; 65: 75-79.

6. Ericson G, Nilson H, Bergman B. Cross-sectional study of patients fitted with fixed partial dentures with special reference to the caries situation. Scand J Dent Res 1990; 98: 8-16.

7. Valderhaug I, Ellingsen JE, Jokstad A. Oral hygiene, periodontal conditions and carious lesions in patients treated with dental bridges. A 15 -year clinical and radiographic follow-up study. J Clin Periodontol 1993; 20: 482-489.

8. Moretti LA, Barros RR, Costa PP, et al. The influence of restorations and prosthetic crowns finishing lines on inflammatory levels after non-surgical periodontal therapy. J Int Acad Periodontol 2011; 13: 65-72.

9. Reitemeier $\mathrm{B}$, Hänsel $\mathrm{K}$, Walter $\mathrm{MH}$, Kastner $\mathrm{C}$, Toutenburg $\mathrm{H}$. Effect of posterior crown margin placement on gingival health. J Prosthet Dent 2002; 87: 167-172.

10. Günay H, Seeger A, Tschernitschek H, Geurtsen W. Placement of the preparation line and periodontal health--a prospective 2-year clinical study. Int J Periodontics Restorative Dent 2000; 20: 171-181.

Evidence-Based Dentistry (2013) 14, 103-104. doi:10.1038/sj.ebd.6400964 